\title{
De novo single-nucleotide and copy number variation in discordant monozygotic twins reveals disease-related genes
}

\author{
Nirmal Vadgama ${ }^{1}$ - Alan Pittman $\mathbb{1}^{1} \cdot$ Michael Simpson $^{2} \cdot$ Niranjanan Nirmalananthan $^{3} \cdot$ Robin Murray $^{4}$ • \\ Takeo Yoshikawa $\mathbb{D}^{5}$ - Peter De Rijk $\oplus^{6}$ - Elliott Rees ${ }^{7} \cdot$ George Kirov $^{7} \cdot$ Deborah Hughes $^{1} \cdot$ Tomas Fitzgerald $^{8}$. \\ Mark Kristiansen ${ }^{9} \cdot$ Kerra Pearce $^{9} \cdot$ Eliza Cerveira $^{10} \cdot$ Qihui Zhu $^{10} \cdot$ Chengsheng Zhang $^{10} \cdot$ Charles Lee $^{10}$. \\ John Hardy ${ }^{1} \cdot$ Jamal Nasir ${ }^{11,12}$
}

Received: 28 September 2018 / Revised: 18 February 2019 / Accepted: 1 March 2019 / Published online: 18 March 2019

(c) European Society of Human Genetics 2019

\begin{abstract}
Recent studies have demonstrated genetic differences between monozygotic (MZ) twins. To test the hypothesis that early post-twinning mutational events associate with phenotypic discordance, we investigated a cohort of 13 twin pairs $(n=26)$ discordant for various clinical phenotypes using whole-exome sequencing and screened for copy number variation (CNV). We identified a de novo variant in PLCB1, a gene involved in the hydrolysis of lipid phosphorus in milk from dairy cows, associated with lactase non-persistence, and a variant in the mitochondrial complex I gene $M T-N D 5$ associated with amyotrophic lateral sclerosis (ALS). We also found somatic variants in multiple genes (TMEM225B, KBTBD3, TUBGCP4, TFIP11) in another MZ twin pair discordant for ALS. Based on the assumption that discordance between twins could be explained by a common variant with variable penetrance or expressivity, we screened the twin samples for known pathogenic variants that are shared and identified a rare deletion overlapping $A R H G A P 11 B$, in the twin pair manifesting with either schizotypal personality disorder or schizophrenia. Parent-offspring trio analysis was implemented for two twin pairs to assess potential association of variants of parental origin with susceptibility to disease. We identified a de novo variant in RASD2 shared by 8-year-old male twins with a suspected diagnosis of autism spectrum disorder (ASD) manifesting as different traits. A de novo CNV duplication was also identified in these twins overlapping CD38, a gene previously implicated in ASD. In twins discordant for Tourette's syndrome, a paternally inherited stop loss variant was detected in $A A D A C$, a known candidate gene for the disorder.
\end{abstract}

Supplementary information The online version of this article (https:// doi.org/10.1038/s41431-019-0376-7) contains supplementary material, which is available to authorised users.

Jamal Nasir

jamal.nasir@northampton.ac.uk

1 Institute of Neurology, University College London, London WC1N 3BG, UK

2 Division of Genetics and Molecular Medicine, King's College London, London, UK

3 St George's University Hospitals NHS Foundation Trust, London SW17 0QT, UK

4 Institute of Psychiatry, Psychology, and Neuroscience, King's College, London, UK

5 RIKEN Brain Science Institute, Wako, Saitama 351-0198, Japan

6 Applied Molecular Genomics Group, University of Antwerp, Antwerp, Belgium

\section{Introduction}

Twin studies have laid the foundation for exploring the genetics of complex traits and common diseases [1], and

7 Centre for Neuropsychiatric Genetics and Genomics, Institute of Psychological Medicine and Clinical Neurosciences, Cardiff University, Cardiff, UK

8 The European Bioinformatics Institute (EMBL-EBI), Cambridge, UK

9 UCL Great Ormond Street Institute of Child Health, London WC1N 1EH, UK

10 Jackson Laboratory for Genomic Medicine, Farmington, CT 06032, USA

11 Cell Biology and Genetics Research Centre, St. George's University of London, London, UK

12 Present address: Molecular Biosciences Research Group, University of Northampton, Northampton NN1 5PH, UK 
heritability estimates for conditions such as schizophrenia (0.79) [2], amyotrophic lateral sclerosis (ALS) (0.61) [3], autism spectrum disorder (ASD) (0.95) [4], and Tourette's syndrome (0.77) [5]. The case co-twin design using identical twins discordant for a trait or disorder has thrown light on the determinants of health and disease and causes of individual differences in normal and abnormal behaviour. Recent advancements in DNA-sequencing techniques has led to significant methodological improvements, allowing the comparison of twin genomes up to the base pair level, which has challenged the assumption that monozygotic (MZ) twins are genetically identical.

The underlying genetic differences between co-twins, including single-nucleotide variants (SNVs), indels, gene conversion, copy number variations (CNVs) and postzygotic mitotic recombination, can arise during embryonic development. These variations have been proposed as potential genetic mechanisms responsible for discordant MZ twins [6]. Therefore, comparing the genomes of discordant MZ twins signifies a promising opportunity for the search for novel variants implicated in disease, which may ultimately narrow the conceptual gap of missing heritability.

Studies of de novo SNVs in parent-offspring trios have identified a mutation rate estimated to range between $(0.82-1.70) \times 10^{-8}$ mutations per base per generation [7-9]. Post-twinning mutations result in somatic mosaicism, a phenomenon defined as two or more genetically distinct populations of cells [10]. Francioli et al. [11] compared 350 validated variants in $\mathrm{MZ}$ twins and found that $\sim 97 \%$ of the variants were germline and $\sim 3 \%$ were somatic. In another study, an important fraction of de novo mutations presumed to be germline in fact occurred either post-zygotically in the offspring, or were inherited because of low-level mosaicism in one of the parents [12]. The mitotic events during embryogenesis, resulting in somatic mosaicism may thus have an important and significant role in human disease and MZ twin discordance. Postzygotic de novo SNVs and CNVs have been found in $\mathrm{MZ}$ twins discordant for a range of disorders, including frontometaphyseal dysplasia [13], Dravet's syndrome [14], Proteus syndrome [15], attention problems [16], and schizophrenia [17],

We performed whole-exome sequencing (WES) and CNV analysis of DNA from thirteen twin pairs discordant for a range of complex disorders. For a twin pair discordant for Tourette's syndrome and another pair where both exhibit signs of ASD, we were able to perform parent-offspring trio analysis using DNA obtained from the parents. With the aim to identify potential genetic factors that influence disease manifestation, it was hypothesised that de novo genetic mechanisms could increase the risk of disease onset. To investigate this hypothesis, the burden of rare SNVs, indels and CNVs overlapping disease-related genes were analysed.
Considering the estimated somatic mutation rate is low and that these variants can be obfuscated by the relatively high error rate of next-generation sequencing (NGS) [18], a highly sensitive filtering method with high specificity, sequence resolution and coverage should be implemented. We provide evidence for de novo SNVs, indels and CNVs in disease-related genes associated with a variety of clinical phenotypes presenting in discordant MZ twins. In addition, the identification of potentially pathogenic variants shared by discordant MZ twins, a possibility that has been overlooked in earlier studies, suggests phenotypic discordance could be explained by a common variant modulated by a secondary genetic or epigenetic mechanism, including variants in regulatory regions or modifier genes.

\section{Materials and methods}

\section{Sample procurement}

Subjects were 13 twin pairs discordant for a range of clinical phenotypes (see Table 1 and Supplementary Material), including the parents of two twin pairs discordant for ASD and Tourette syndrome. DNA samples of five of the twin pairs were obtained from the Coriell Cell Repository (https://coriell.org/). Peripheral blood and/or buccal samples were collected from subjects recruited into the study after obtaining written informed consent.

\section{Whole-exome sequencing}

WES libraries were prepared with Agilent SureSelect V6 and sequenced on an Illumina HiSeq3000 using a 75-bp paired-end reads protocol (see Supplementary Material).

\section{Sequencing data analysis}

We have an in-house set of $\sim 6000$ exomes (from controls and rare diseases) for cross-checking any shortlisted candidate variants, and sequencing artefacts. Sequence alignment to the human reference genome (UCSC hg19), and variant calling and annotation were performed with our in-house pipeline. In brief, this involves alignment with NovoAlign, removal of PCR-duplicates with Picard Tools followed by (sample-paired) local realignment around indels and germline variant calling with HaplotypeCaller according to the Genome Analysis Toolkit (GATK) best practices [19].

Mosaic variants were identified with GATK MuTect2 (version 2.0) and VarScan2 (version 2.4.3), using each pair as reference to one-another. The raw list of SNVs and indels were then filtered using ANNOVAR [20]. Variants in splicing regions, 5'-UTR, 3'-UTR, and protein-coding 
Table 1 Clinical characteristics and demographic information on the MZ twin cohort

\begin{tabular}{|c|c|c|c|c|c|c|c|}
\hline Subject & Status & Diagnosis & Sex & Age & Age of onset & Ethnicity & DNA source \\
\hline LAS & Proband & ALS & $\mathrm{F}$ & Died at 71 & 67 & Caucasian & Saliva \\
\hline SUS & $\mathrm{MZ}$ twin & - & $\mathrm{F}$ & 75 & - & Caucasian & Saliva \\
\hline 218 & Proband & ALS & M & 54 & 50 & Caucasian & LCL \\
\hline 318 & MZ twin & - & M & 54 & - & Caucasian & LCL \\
\hline 421 & Proband & ALS & $\mathrm{F}$ & 58 & 55 & Caucasian & LCL \\
\hline 422 & MZ twin & - & $\mathrm{F}$ & 58 & - & Caucasian & LCL \\
\hline 242 & Proband & ALS & M & 35 & 34 & Caucasian & LCL \\
\hline 243 & MZ twin & - & M & 35 & - & Caucasian & LCL \\
\hline $\mathrm{KG}(\mathrm{s})$ & Proband & Ischaemic stroke & $\mathrm{F}$ & 62 & 56 & Caucasian & Saliva \\
\hline $\mathrm{KG}(\mathrm{b})$ & & & & & & & Blood \\
\hline $\mathrm{HG}(\mathrm{s})$ & MZ twin & - & $\mathrm{F}$ & 62 & - & Caucasian & Saliva \\
\hline $\mathrm{HG}(\mathrm{b})$ & & & & & & & Blood \\
\hline KEL & Proband & LNP & $\mathrm{F}$ & 23 & 5 & Caucasian & Saliva \\
\hline KIR & MZ twin & - & $\mathrm{F}$ & 23 & - & Caucasian & Saliva \\
\hline $\mathrm{AFF}$ & Proband & IBM & M & 68 & 66 & Caucasian & Blood \\
\hline UNAFF & MZ twin & - & M & 68 & - & Caucasian & Blood \\
\hline DS & Father & - & M & UN & - & Caucasian & Saliva \\
\hline DV & Mother & - & $\mathrm{F}$ & UN & - & Afro-Caribbean & Saliva \\
\hline $\mathrm{RP}$ & Proband & ASD & M & 10 & 7 & Mixed & Saliva \\
\hline $\mathrm{OH}$ & MZ twin & ASD? & M & 10 & - & Mixed & Saliva \\
\hline 487 & Father & TS, OCD, ADHD & M & 44 & 6 & American Indian & Blood \\
\hline 488 & Mother & - & $\mathrm{F}$ & 44 & - & Caucasian & Blood \\
\hline 489 & $\mathrm{MZ}$ twin & - & M & 15 & - & Mixed & Blood \\
\hline 490 & Proband & TS & M & 15 & 7 & Mixed & Blood \\
\hline PD821 & Proband & PD & M & 40 & 30 & Caucasian & Blood \\
\hline PD161 & MZ twin & - & M & 40 & - & Caucasian & Blood \\
\hline $\mathrm{VF}$ & Proband & HSP & $\mathrm{F}$ & 46 & 34 & Caucasian & Blood \\
\hline $\mathrm{LF}$ & MZ twin & HSP? & $\mathrm{F}$ & 46 & - & Caucasian & Blood \\
\hline RT1a & $\mathrm{MZ}$ twin & SCPD & M & UN & UN & Asian & Blood \\
\hline RT1b & Proband & SCZ & M & UN & UN & Asian & Blood \\
\hline IP16 & Proband & $\mathrm{SCZ}$ & M & UN & UN & Caucasian & Saliva \\
\hline IP17 & $\mathrm{MZ}$ twin & - & M & UN & - & Caucasian & Saliva \\
\hline
\end{tabular}

$A D H D$ Attention deficit hyperactivity disorder, $A L S$ amyotrophic lateral sclerosis, $A S D$ Autism spectrum disorder, $H S P$ hereditary spastic paraplegia, $I B M$ inclusion body myositis, $L N P$ lactase non-persistence, $O C D$ obsessive compulsive disorder, $P D$ Parkinson's disease, SCPD schizotypal personality disorder, $S C Z$ schizophrenia, TS Tourette's syndrome, UN unknown regions, such as missense, frameshift, stop loss, and stop gain mutations, were considered. Priority was given to rare variants $(<1 \%$ in public databases, including NHLBI Exome Variant Server, Complete Genomics 69 (cg69), Exome Aggregation Consortium and 1000 Genomes). In silico prediction of pathogenicity was assessed using SIFT [21], PolyPhen2 [22], and MutationTaster [23]. Conservation of nucleotides was scored using Genomic Evolutionary Rate Profiling [24]. For the parent-offspring trio analyses and detection of shared variants, joint genotyping was performed on all samples $(n=32)$. False-positive variants were removed based on the Variant Quality Score Recalibration (tranche sensitivity <99.00), including low quality variants that had low depth of coverage $(<10)$ and poor genotype quality $(<20)$. Potentially pathogenic variants were submitted to the variant database LOVD (https://databases. lovd.nl/shared) submission IDs 00207897-00207091 and 00210051-00210057.

\section{Variant validation by Sanger sequencing}

DNAs were amplified by PCR, using primers specific to the resulting discordant indels and SNVs (Table 2). Sanger sequencing was performed on an ABI 3730XL Genetic Analyzer (PE Applied Biosystems, Forest City, CA, USA) to validate the variants. Forward and reverse primer 


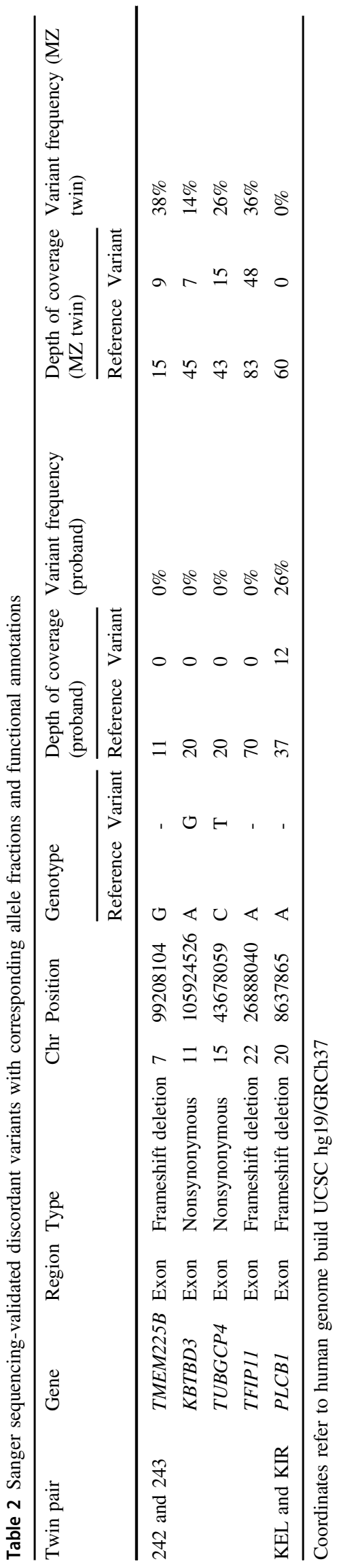

sequences for the candidate loci are listed in Supplementary Table 3.

\section{Genome-wide SNP genotyping}

Genotyping was performed according to the manufacturer's instructions using the Illumina HumanCore v12 BeadChip (Illumina Inc., San Diego, CA, USA). In the quality control, sample sex, and twin zygosity were genotypically confirmed, and no samples were excluded based on a genotyping call rate threshold of $>0.997$.

\section{Copy number variant detection}

Log R Ratios and B-allele frequencies were generated using Illumina GenomeStudio software (v2011.1) and used to call CNVs with PennCNV [25]. CNV calling was performed following the standard protocol and adjusting for GC content. CNVs were then excluded if they were covered by $<3$ probes. After $\mathrm{CNV}$ merging, the remaining $\mathrm{CNV}$ s were visually re-evaluated using the GenomeStudio genotyping module. cnvPartition was used as the secondary CNV detection algorithm using the default parameters. All CNV coordinates are according to UCSC build 37/hg19.

\section{Computational validation by ExomeDepth}

CNVs, called by PennCNV and cnvPartition, were corroborated by computational validation with ExomeDepth. CN calls that were shared by all three calling algorithms (PennCNV, cnvPartition, and ExomeDepth) were considered high-confidence CNVs.

The read count information was extracted from the individual BAM files using the R package Rsamtools. All reads were paired-end. Only reads with a Phred scaled mapping quality $\geq 20$, distance of $<1000 \mathrm{bp}$ from each other and in the correct orientation, were included. The location was defined by the middle location between the extreme ends of both paired reads. Exons closer than $50 \mathrm{bp}$ were merged into a single location owing to the inability to properly separate reads mapping to either of them. Parameters for ExomeDepth were applied according to the instructions provided by the user guide.

\section{Results}

\section{Identifying discordant variants}

WES data were analysed by VarScan2 and MuTect2, using the annotated variant and genotype attained by the HaplotypeCaller-based analysis as reference, to explore the possible occurrence of low-frequency variants compatible 


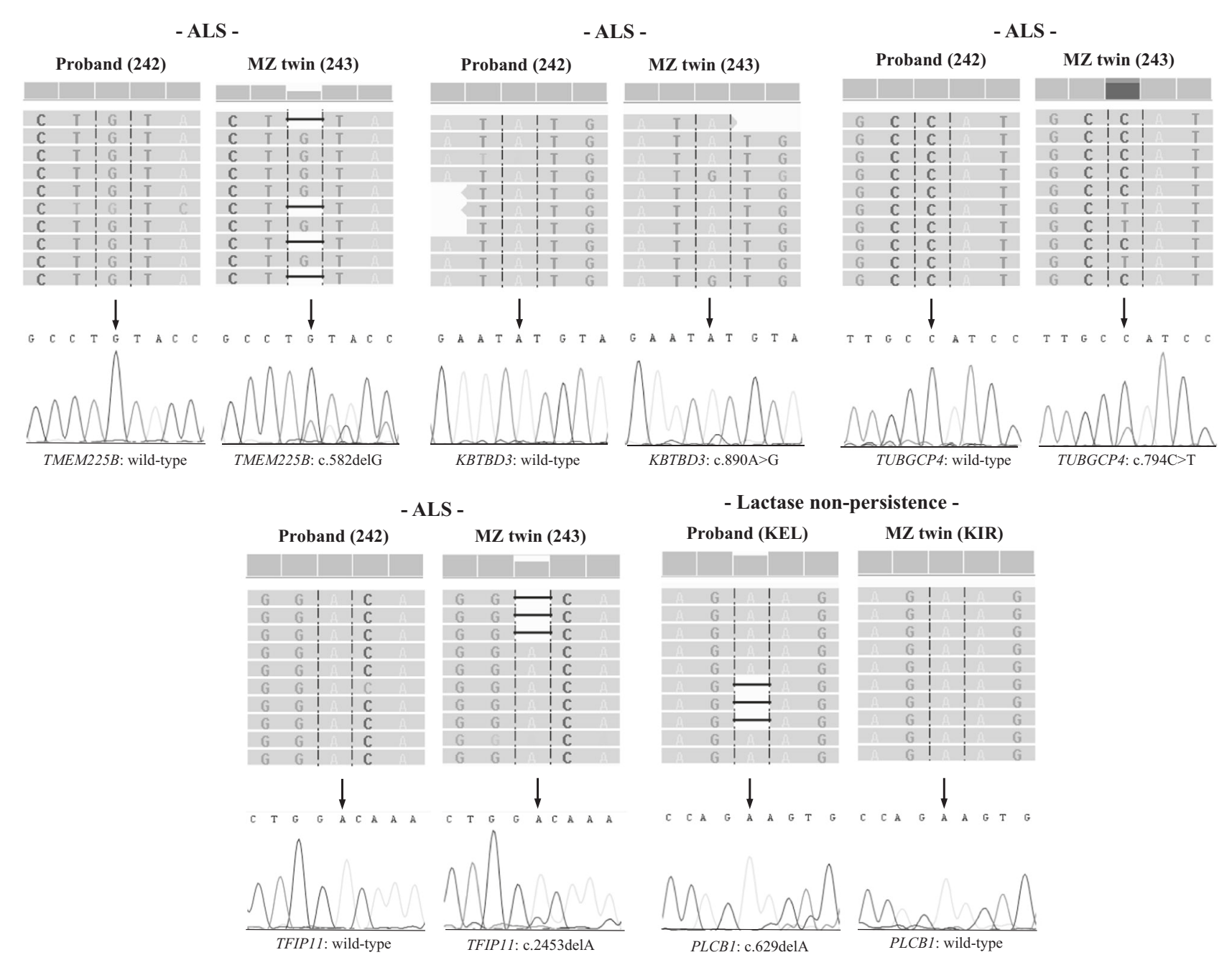

Fig. 1 IGV screenshots and electropherograms confirming somatic variation in twins discordant for ALS (242 and 243) and lactase non-persistence (KEL and KIR)

with a mosaicism state. As there is a possibility of the unaffected twin having a de novo variant that is not present in the affected twin, a reverse pairwise analysis was also performed where the affected twin was classified as the "normal" sample and unaffected twin as the "tumour" sample (Supplementary Tables 4 and 5). The resulting discordant variants were further filtered by excluding those variants that were likely to be non-functional, e.g., synonymous variants and/or variants outside the exonic regions. There are exceptions to this rule, such as for the twin pair discordant for lactase non-persistence (KEL and KIR), where causally linked variants have been reported in intronic regions, with an MAF > 0.01 [26].

After applying our stringent filtering criteria, 20 putative discordant variants were identified across all 13 twin pairs (Supplementary Table 6). However, only five of these variants could be validated with Sanger sequencing (Table 2), including a somatic variant in the $P L C B 1$ gene in the twin affected with lactase non-persistence (KEL and
KIR) and variants in four genes (TMEM225B, KBTBD3, TUBGCP4, TFIP11) in the unaffected twin of an ALSdiscordant pair (242 and 243 (Fig. 1). The success rate of $20 \%$ is due in part to the limited sensitivity of Sanger sequencing to detect alleles with a frequency of $<15 \%$ [27]. A comparison of saliva and blood tissues for the twin pair discordant for ischaemic stroke did not reveal any evidence of somatic mosaicism.

\section{Identifying shared function-altering variants}

To test the hypothesis that the same rare, dominant or recessive variants could contribute to the phenotype for given twin pairs where no discordant variant was found, all potentially damaging shared exonic variants were examined, including known disease-associated loci that could explain disease onset according to a model taking into account the possibility of incomplete penetrance or variable expressivity. 
After application of the filtering criteria (see Materials and Methods), each twin pair shared 200 rare variants that are predicated to affect protein function. These were screened against lists of disease-specific susceptibility genes, which were obtained from various databases, including PubMed, OMIM, NIH GTR, DisGeNET, DISEASES, ALSoD, ALSGene, PDGene, SZDB, and SZGene. This produced a total of 113 variants for the entire cohort. These variants were further reduced by removing those found in multiple other samples, repetitive sequences, or systematic mismapping of paralogous sequences. Variants that were unambiguous upon manual inspection in Integrative Genomics Viewer (IGV) were retained. In total, 23 shared variants were identified in known diseasesusceptibility genes. These variants with their functional categories are shown in Table 3. Considering that the shared variants between co-twins were absent in all other samples, it would be extremely unlikely to obtain false positives in the same gene location in both twin siblings.

\section{Parent-offspring trio analysis}

The availability of parental DNAs for two pairs of twins discordant for ASD and Tourette's syndrome, respectively, allowed us to further investigate the origin of the shared variants. A total of 217,290 variants were called in GATK's joint analysis in the entire cohort. Variants shared by the MZ twins, but absent in their parents' blood samples and in the other twins, were considered to be de novo germline variants of parental origin. After applying these initial exclusion criteria, a total of 424 and 412 putative de novo SNVs and indels were detected in the two twin pairs discordant for ASD and Tourette's syndrome, respectively. These variants were filtered using the same parameters set for postzygotic de novo variant detection. Upon manual review in $\mathrm{IGV}$, variants could be further excluded on the basis that they were miscalled in one of the parents.

A nonsynonymous variant in $R A S D 2$, a gene encoding for a GTP-binding protein Rhes on chromosome 22 (NM_014310.3:c.170 G > A:p.(Arg57His)), was found in the twins with ASD discordant for severity. This variant is not reported in the dbSNP, 1000 Genomes, cg69 nor in the in-house database of 6000 exomes. In the ExAC database containing $>60,000$ human exome data, the variant was found with an allele frequency of 8.13E-06 in the total population (allele count of 1/121112). The variant is also highly conserved across multiple species and predicted to be deleterious in online available bioinformatics tools [21-23].

No shared de novo variant of parental origin was detected in the twins discordant for Tourette's syndrome after application of our stringent filtering criteria described above. Nevertheless, as the father of the twins also had the condition, it is likely that both twins had inherited variants associated with the disorder. We focused our attention on variants consistent with a dominant mode of inheritancenamely, variants that are homozygous or heterozygous in the affected father, absent in the mother, and heterozygous in the twins.

After filtering for rare or novel variants that were predicted to be damaging by at least one of the pathogenicity prediction tools led to the identification of 41 variants inherited from the father. Only one of these variants have previously been implicated in Tourette's syndrome, from our comprehensive list of 138 genes mined from various databases and search of literature. This stop loss variant in $A A D A C$, a gene encoding for arylacetamide deacetylase on chromosome 3 (NM_001086.2:c.1198 T > C:p. $(* 400$ Glnext*1)), plus the de novo variant associated with ASD, were validated with Sanger sequencing in the two families (Fig. 2).

\section{Mitochondrial DNA analysis}

We next tested the hypothesis that different levels of mtDNA heteroplasmy might account for the phenotypic discordance between the twins. After applying a minimum read count threshold of 10 , a total of 399 shared and discordant variants were identified between the twins. These variants included 34 heteroplasmic variants and 365 homoplasmic variants. A total of 36 variants unique to either the affected or unaffected twin were verified using IGV. Among these, 23 were distributed on 12 genes throughout the mitochondrial genome, and 8 were localised at the hypervariable segments HV1 (16024-16383) and HV2 (57-372). Most of the discordant variants came from the twin pair 421 and 422. These samples were excluded from further analysis due to the likelihood of artefacts created from high passage transformed immortalised cell lines. Variants in hypervariable segments were also removed. A novel nonsynonymous variant in MT-ND5 (m.1260 C > A:p.(Ser420Arg)), the complex I mitochondrial gene, was detected in the unaffected twin SUS, but was absent in the co-twin affected with ALS, suggesting it might play a protective role. Although this discordant variant had a high depth of coverage (with the number of reads ranging from 130 to 220), it could not be excluded or confirmed with Sanger sequencing owing to a low allele fraction of $9 \%$.

\section{Copy number variant analysis}

CNVs were initially called in PennCNV if they are covered by $\geq 3$ probes in order to detect small CNVs that would potentially be filtered out of the data. As this is expected to result in a higher frequency of false positive calls, CNVs were also called using cnvPartition, and $\mathrm{CN}$ segments were only included in further analysis if the $\mathrm{CN}$ calls agreed 


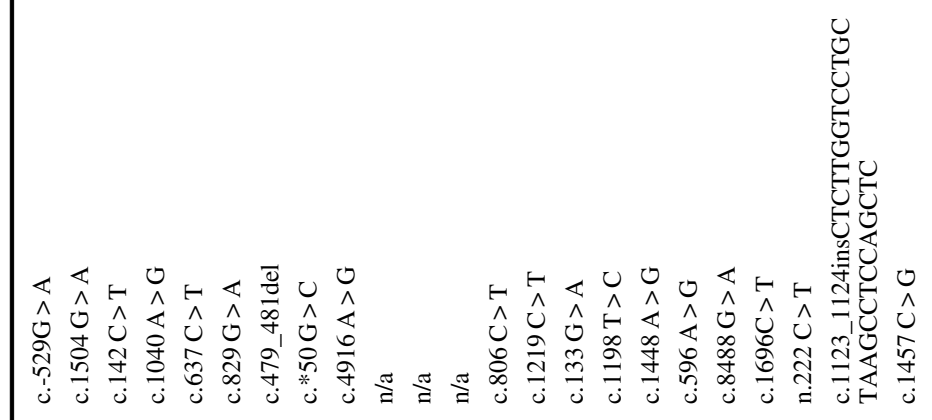

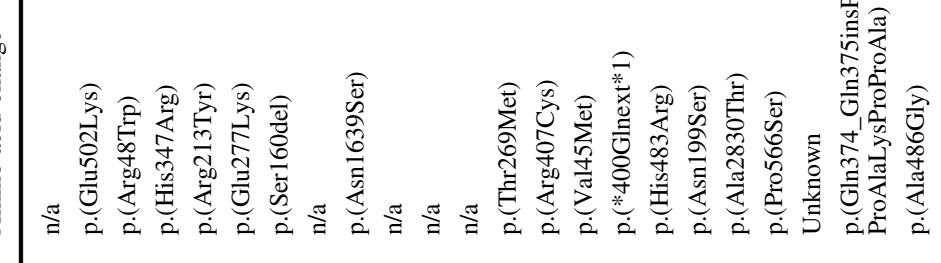

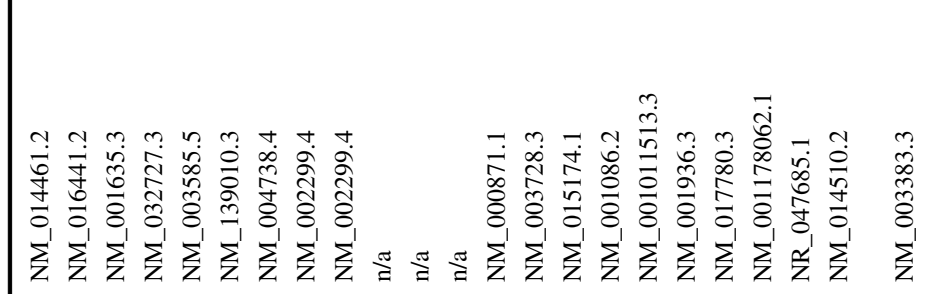

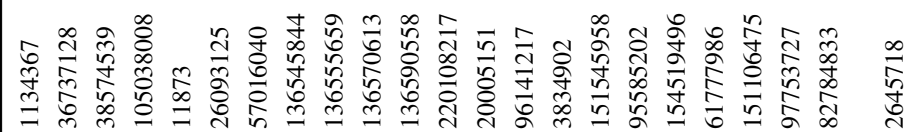


Fig. 2 IGV screenshots and electropherograms confirming a germline de novo and inherited variants. a A parental germline de novo variant in $R A S D 2$ in twins with behavioural issues and ASD (OH and RP), which is absent in their parents (DS and DV). b A stop loss variant detected in $A A D A C$ in twins discordant for Tourette's syndrome, inherited from the affected father $\mathbf{a}$

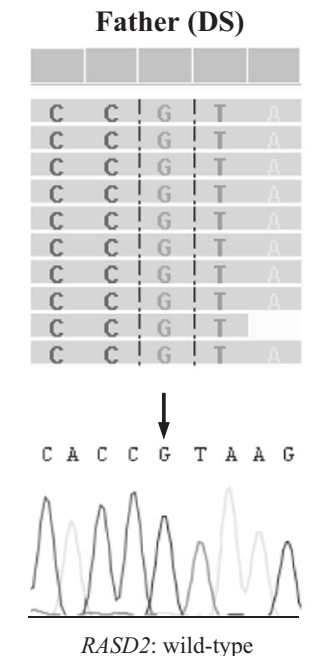

b

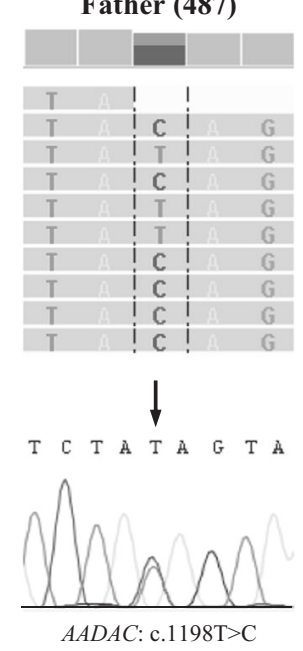

- ASD -

\begin{tabular}{|l|l|l|l|l}
\multicolumn{5}{c|}{ Mother (DV) } \\
\hline \multicolumn{1}{|c|}{} \\
\hline C & C C & G & T \\
\hline C & C & G & T \\
\hline C & C & G & T \\
C & C & G & T \\
\hline C & C & G & T \\
C & C & G & T \\
C & C & G & T \\
C & C & G & T \\
C & C & G & T \\
C & C & G & T \\
\hline
\end{tabular}

Proband (RP)

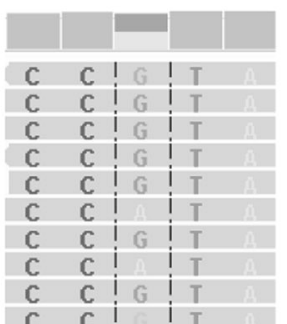

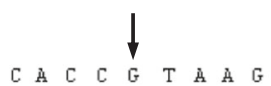

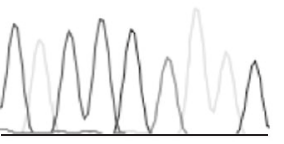

RASD2: wild-type

RASD2: c. 170G $>\mathrm{A}$
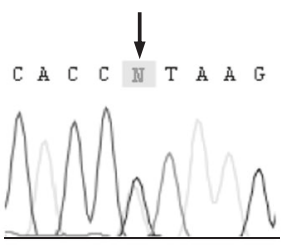

MZ twin (OH)
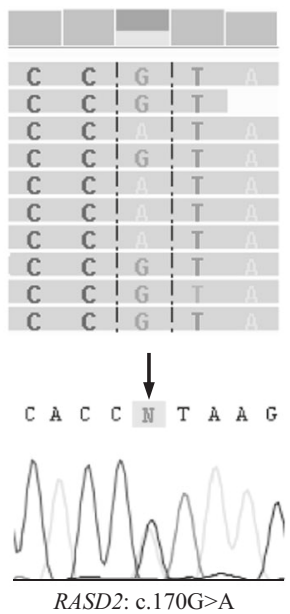

MZ twin (489)

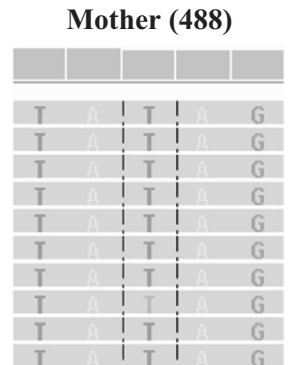

Proband (490)
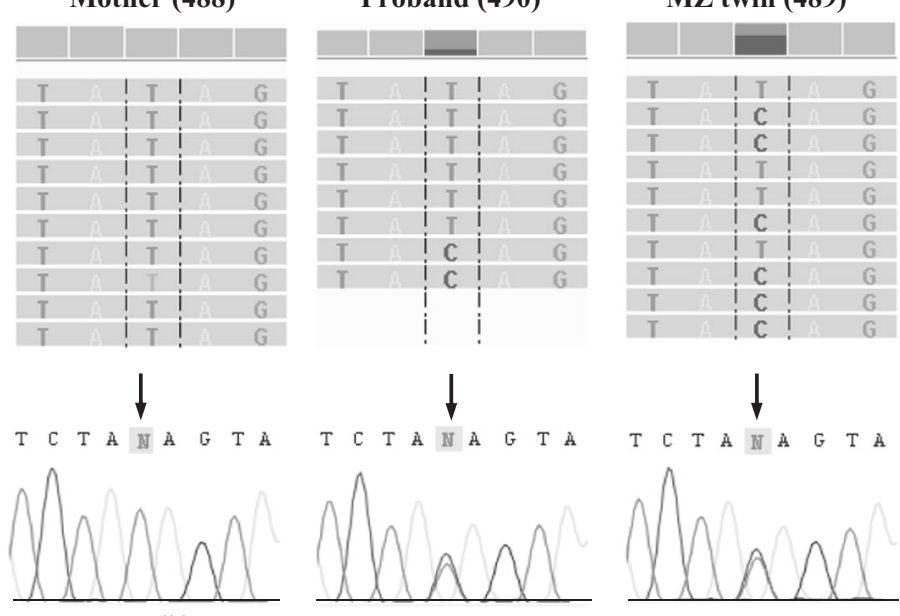

$A A D A C:$ c. $1198 \mathrm{~T}>\mathrm{C}$
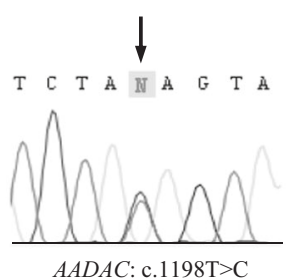

between both algorithms. The results obtained from SNP array analysis are summarised in Supplementary Table 7. Putative CNVs were further confirmed against WES CNV calls using ExomeDepth. $\mathrm{CN}$ calls that were shared by all three calling algorithms were considered for downstream analysis (Table 4).

For the CNVs shared between co-twins, we focused on subsets of genes that are associated with known phenotypes in disease databases such as OMIM and DisGeNET, or genes that are intolerant to LoF variants based on the Residual Variation Intolerance Score (RVIS) or the probability of being loss-of-function intolerant (pLI) [28]. An RVIS $<0.0$ means that a given gene has less common functional variation than expected, and is referred to as "intolerant", whereas an RVIS $>0.0$ indicates that a gene has more common functional variation than expected. Genes with high pLI scores (pLI $\geq 0.9)$ are extremely LoF intolerant, whereas genes with low pLI scores $(\mathrm{pLI} \leq 0.1)$ are LoF tolerant (Table 4).

No CNV differences between co-twins or tissue types were found. However, four CNVs of parental germline origin were identified by the $\mathrm{CN}$ calling algorithms used but were not experimentally validated. Three were found to not overlap any genes or regulatory regions, and one was a CNV duplication found in the twins exhibiting signs of ASD (OH and RP) but differing in severity and overlapping $>85 \%$ proximal of $C D 38$. This shared de novo CNV was also called by ExomeDepth.

The $138 \mathrm{~kb}$ deletion in 15q13.2 spanning ARHGAP11B in the schizophrenia-discordant twins RT1a/RT1b was of particular interest as gene ontology terms include cerebral cortex development. $\mathrm{CNV}$ deletions containing $A R H-$ GAP11B have previously been associated with schizophrenia [29] in numerous studies. The twins are discordant 
Table 4 Genes within CNV regions identified by PennCNV, cnvPartition, and ExomeDepth

\begin{tabular}{|c|c|c|c|c|c|c|c|}
\hline $\begin{array}{l}\text { Diagnosis of } \\
\text { affected twin }\end{array}$ & Gene & $\begin{array}{l}\text { Copy number } \\
(1=\text { deletion; } \\
2=\text { duplication })\end{array}$ & Description & $\begin{array}{l}\text { Disease association } \\
\text { (DisGeNET and DISEASES) }\end{array}$ & RVIS & pLI & $\% \mathrm{HI}$ \\
\hline ALS & $L A R G E 1$ & 2 & $\begin{array}{l}\text { LARGE xylosyl- and } \\
\text { glucuronyltransferase } 1\end{array}$ & $\begin{array}{l}\text { Congenital muscular dystrophy; } \\
\text { mental retardation syndromes; }\end{array}$ & $-1.11(10.98 \%)$ & 0.96 & 4.10 \\
\hline HSP & $S L C 8 A 1$ & 1 & Solute carrier family 8 member A1 & $\begin{array}{l}\text { Myocardial ischaemia; status } \\
\text { epilepticus }\end{array}$ & $-1.41(6.88 \%)$ & 0.99 & 6.09 \\
\hline Ischaemic stroke & ELOVL6 & 2 & ELOVL fatty acid elongase 6 & $\begin{array}{l}\text { Heart failure; Psoriasis; obesity; } \\
\text { atherosclerosis; atopic dermatitis }\end{array}$ & $-0.27(36.97 \%)$ & 0.97 & 3.68 \\
\hline \multirow[t]{3}{*}{ Lactose intolerance } & $M B D 3 L 2$ & 2 & $\begin{array}{l}\text { Methyl-CpG binding domain } \\
\text { protein } 3 \text { like } 2\end{array}$ & Stomach neoplasms & $\mathrm{n} / \mathrm{a}$ & $\mathrm{n} / \mathrm{a}$ & 95.74 \\
\hline & ADGRE4P & 2 & $\begin{array}{l}\text { Adhesion } \mathrm{G} \text { protein-coupled } \\
\text { receptor E4, pseudogene }\end{array}$ & Colorectal cancer & $\mathrm{n} / \mathrm{a}$ & $\mathrm{n} / \mathrm{a}$ & $\mathrm{n} / \mathrm{a}$ \\
\hline & ADGRE1 & 2 & $\begin{array}{l}\text { Adhesion G protein-coupled } \\
\text { receptor E1 }\end{array}$ & $\begin{array}{l}\text { Liver cirrhosis; secondary } \\
\text { periodontitis }\end{array}$ & $2.21(97.76 \%)$ & 0.00 & 90.69 \\
\hline ASD & CD38 & 2 & CD38 molecule & Autism spectrum disorders & $0.47(70.44 \%)$ & 0.00 & 77.57 \\
\hline Tourette's syndrome & ТОР $3 B$ & 2 & DNA topoisomerase III beta & $\begin{array}{l}\text { Cognitive impairment; } \\
\text { schizophrenia }\end{array}$ & $-0.34(33.91 \%)$ & 0.11 & 15.86 \\
\hline $\begin{array}{l}\text { Tourette's syndrome/ } \\
\text { Parkinson's disease }\end{array}$ & $N L G N 1$ & 2 & Neuroligin 1 & $\begin{array}{l}\text { Autistic disorder; schizophrenia; } \\
\text { bipolar disorder; depression; } \\
\text { memory impairment }\end{array}$ & $-1.26(8.92 \%)$ & 0.76 & 1.37 \\
\hline \multirow[t]{2}{*}{$\begin{array}{l}\text { Inclusion body } \\
\text { myositis }\end{array}$} & AUTS2 & 2 & $\begin{array}{l}\text { AUTS2, activator of transcription } \\
\text { and developmental regulator }\end{array}$ & $\begin{array}{l}\text { Neurological disorders; acute } \\
\text { lymphoblastic leukaemia; aging of } \\
\text { the skin; early-onset androgenetic } \\
\text { alopecia; cancer }\end{array}$ & $-1.94(3.32 \%)$ & 1.00 & 0.51 \\
\hline & LILRB3 & 2 & $\begin{array}{l}\text { Leukocyte immunoglobulin like } \\
\text { receptor B3 }\end{array}$ & Lung diseases; Takayasu's arteritis & $0.79(81.49 \%)$ & 0.00 & 97.17 \\
\hline \multirow[t]{2}{*}{ Schizophrenia } & $A R H G A P I I B$ & 1 & $\begin{array}{l}\text { Rho GTPase-activating protein } \\
\text { 11B }\end{array}$ & $\begin{array}{l}\text { 15q13.3 microdeletion syndrome; } \\
\text { schizophrenia; bipolar disorder }\end{array}$ & $0.07(52.75 \%)$ & 0.07 & 81.20 \\
\hline & ARHGAP5 & 2 & Rho GTPase-activating protein 5 & Liver carcinoma; breast cancer & $-1.31(8.10 \%)$ & 0.99 & 8.15 \\
\hline
\end{tabular}

Genes that are in the RefSeq database (http://www.ncib.nlm.nih.gov/gene) and Ensembl database (http://www.ensembl.org) are reported. RVIS v4 is constructed on the ExAC v2 data release. Web resources DisGeNET and DISEASES were used to gather disease-gene associations from various expert curated databases and text-mining derived associations

as the co-twin of the proband was diagnosed with schizotypal personality disorder.

\section{Discussion}

We report the successful detection of genomic differences between phenotypically discordant MZ twins. Numerous studies have failed to find somatic variants using NGS technology between discordant twins, with variants often masked by a substantial number of false positives. Such studies include twins discordant for multiple sclerosis [30], Crohn's disease [31], congenital hypothyroidism [32], and ALS [33]. By taking a union of MuTect2 and VarScan2 to identify somatic variants, we offer a proof of concept for assessing the genetic aetiology of complex traits in discordant twins. A comparison of variant detection tools showed that MuTect 2 identifies more low coverage somatic variants and has excellent capability in both control of false calls and discovery of potential true positives. VarScan2 was as efficient in low-frequency variants detection but exhibited an advantage in discovering somatic SNVs with relatively high frequencies, which makes it a beneficial supplement of MuTect2 [34].

Our data suggest new variants are relatively common and involve a variety of pre- and postzygotic mechanisms affecting nuclear and mitochondrial genes, including epistasis. For some of the twin pairs, no discordant variants were identified, and the shared variants present in diseasesusceptibility genes do not readily explain the twins' discordant phenotypes. We postulate discordance could be explained by shared pathogenetic variants resulting in incomplete penetrance or variable expressivity followed by secondary genetic or epigenetic changes affecting gene regulation. Other potential contributory factors might involve tissue-specific somatic mutations.

\section{Discordant single nucleotide and indel variants}

A somatic frameshift deletion in $P L C B 1$ was detected in a buccal sample, containing epithelial cells of common developmental origin to the cells lining the gut, derived from the affected twin with lactase non-persistence. PLCB1 is highly expressed in the cardia and colon, and in dairy cows this protein has been shown to hydrolyse most of the lipid phosphorus in the low- and high-density lipoprotein fractions of milk [35]. However, the role of this gene in digestive system disorders remains unclear, but warrants further investigations to verify the significance of this variant, if any, in lactase non-persistence.

In the ALS-discordant pair, two discordant nonsynonymous variants were identified in $K B T B D 3$ and $T U B G C P 4$, and two frameshift deletions in TMEM225B and TFIP11 (Table 2). Although these variants were predicted to disrupt 
protein function, this cannot be reconciled with the fact that they were detected in the unaffected twin. Nevertheless, it is possible that these somatic variants contributed to the phenotypic discordance by having protective effects in the unaffected twin. It is important to note that the DNA samples used for this pair are lymphoblastoid cell lines (LCL)derived, and de novo mutations are known to be caused by the cell line transformation and culturing. However, several studies have suggested low-passage LCLs to be an appropriate representation of the donor's genome [36]. Nevertheless, we acknowledge that independent validation on DNA from uncultured sources is ideal.

\section{Shared single-nucleotide and indel variants}

We examined shared variants with predicted pathogenicity. This included rare homozygous and heterozygous variants, and those in known disease-susceptibility genes.

\section{De novo variant detection in parent-offspring trio analysis}

\section{Autism spectrum disorder}

A nonsynonymous variant (NM_014310.3:c.170 G > A:p. (Arg57His)) of parental germline origin within the RASD family member 2 (RASD2) gene was found in both twins with a suspected diagnosis of ASD and behavioural problems (OH and RP) but showing different degrees of severity. RASD2 belongs to the Ras superfamily of small GTPases and is enriched in the striatum and involved in the modulation of dopaminergic neurotransmission [37]. RASD2 is located on chromosome $22 \mathrm{q} 12.3$, a region that harbours numerous susceptibility loci for psychosis [38], and has been suggested to be a vulnerability gene for neuropsychologically defined subgroups of schizophrenic patients [39]. At present, the co-twin has not been diagnosed but anecdotally has been showing clinical features of ASD.

\section{Tourette's syndrome}

No function-altering germline de novo variants were identified in the twin pair. However, a shared novel stop loss variant in $A A D A C$, a gene encoding for arylacetamide deacetylase on chromosome 3 (NM_001086.2:c.1198 T > C:p.(*400Glnext*1)), was inherited from the father. In a meta-analysis of 1181 patients and 118,730 control subjects, Bertelsen et al. [40] determined a significant association between $A A D A C$ and Tourette's syndrome. Further, functional studies demonstrated that $A A D A C$ is expressed in several brain regions previously implicated in the pathophysiology of Tourette's syndrome, including the Purkinje cell layer of the human cerebellum [40]. CNVs overlapping
$A A D A C$ are the first to be successfully associated with Tourette's syndrome. More recently, Yuan et al. [41] found that variants in $A A D A C$ may be a candidate factor for Tourette's syndrome development in a Han Chinese cohort.

Transcriptome profiling data from The BrainSpan Atlas of the Developing Human Brain (http://www.brainspan.org) illustrates that $A A D A C$ expression peaks in the striatum between birth and adolescence. This is consistent with the typical clinical time course of tic onset, and indeed the age of onset of Tourette's syndrome in the father and the affected twin investigated herein. However, other mechanisms such as epigenetics must be considered to explain the asymptomatic twin. Considering the above evidence, the stop loss variant detected in the father and twins warrants functional studies to investigate the role of $A A D A C$ in the pathogenesis of this disorder.

\section{Copy number shared variants}

A shared de novo CNV duplication of parental germline origin was detected in twins with a suspected diagnosis of ASD (discordant for severity), overlapping CD38, a gene implicated in ADHD [42], social memory, amnesia, and ASD [43]. Although our results do not prove CNV contribution to phenotypic $\mathrm{MZ}$ discordance, the pre-twinning structural events detected in this twin cohort could represent a susceptible genetic background (Table 4).

\section{Schizophrenia}

A deletion in $A R H G A P 11 B(\mathrm{CN}=1)$ and a duplication in ARHGAP5 $(\mathrm{CN}=3)$ were identified in schizophreniadiscordant twin pairs RT1a/RT1b and IP16/IP17, respectively (Supplementary Figures 7 and 8). Although RT1b was diagnosed with schizophrenia, his co-twin (RT1a) had schizotypal personality features.

The ARHGAP5 gene product (a GTPase-activating protein for Rho family members) is linked to Ras, and thus to EGF receptor-mediated proliferation, migration, and differentiation of forebrain progenitors [44]. Therefore, an ARHGAP5 duplication in an $\mathrm{MZ}$ twin pair discordant for schizophrenia might point to an aetiological basis, because schizophrenia has been linked to altered prenatal neurogenesis of cortical neurons [45]. In addition, ARHGAP5 and ARHGAP11B are contained within regions $14 \mathrm{q} 12$ and $15 q 13.2$, respectively, which have previously been associated with schizophrenia [46, 47].

ARHGAP11B resides on chromosome $15 \mathrm{q} 13.2$, one of the most complex and unstable loci in the human genome. Several neurodevelopmental disorders have been linked to structural variants in this and nearby regions [48]. $A R H$ $G A P 11 B$ arose from partial duplication of $A R H G A P 11 A$ in the human lineage, approximately one million years after 
divergence from chimpanzees, but before divergence from Neanderthals [49]. This led to the formation of large and complex human-specific segmental duplications, mediating recurrent rearrangements contributing to $15 \mathrm{q} 13.3$ microdeletion syndrome associated with intellectual disability, epilepsy, and schizophrenia [50]. ARHGAP11B is, to date, the only human-specific gene shown to promote basal progenitor generation and proliferation, including cortical plate augmentation and gyrification induction, and has been proposed to play an important role in the evolutionary expansion of the human neocortex [49].

The duplicated eight exons of ARHGAP11A are almost identical to the paralogous sequence of $A R H G A P 11 B$, and thus not completely queried in high-throughput genetic studies at the same locus and may have been mixed complex rearrangements. Indeed, variations in this region have flown below the radar of available genome-wide technologies, which likely has downplayed its hypothesised associations with neurodevelopmental disorders. Because of the genomic complexity of the region, the extent of human structural diversity and breakpoints of most rearrangement events are poorly understood at the molecular genetic level. Moreover, the wide expression of ARHGAP11B, its multiple functions and modes of regulation - not to mention its absence in other species-present challenges for its study in disease.

Several RhoGAPs have been linked to schizophrenia. For example, a study reported an association between variation in ARHGAP32, which encodes a neuronassociated GTPase-activating protein, and schizophrenia, and schizotypal personality traits [51]. ARHGAP33 regulates synapse development and autistic-like behaviour [52]. A missense polymorphism in ARHGAP3 has been associated with schizophrenia in men [53]. Further, in a genome-wide association study from the Han Chinese population, Wong et al. [54] identified a schizophrenia susceptibility locus on $\mathrm{Xq} 28$, which harbours the gene ARHGAP4. This study shows segmental duplications play an important role in normal variation as well as in genomic disease defining hotspots of rearrangement that are susceptible to variation among the normal population. Considering the above findings, we propose that both ARHGAP5 and ARHGAP $11 B$ are potentially associated with neuropsychiatric disorders, and this preliminary study provides the necessary baseline to begin future studies on disease populations.

\section{Conclusion}

We have successfully identified pre- and postzygotic variants in twin pairs discordant for complex traits. Parentoffspring trio analysis revealed a novel candidate gene for
ASD and a novel variant in a gene implicated in Tourette's syndrome.

This study also sheds new light on the genetics of complex disorders including lactase non-persistence, ALS, and schizophrenia. We show that de novo variants are relatively common, could involve multiple genes, and invoke multiple mechanisms affecting both nuclear and mitochondrial genes. We also show that discordant MZ twins may share common underlying variants and postulate that additional genetic events, such as epigenetic changes, might lead to phenotypic discordance. Thus, previous studies, mostly based on the premise of a de novo mutation in the affected twin, may have missed the opportunity to detect shared variants originating from the parents. Of note, we document a shared pathogenic hexanucleotide repeat expansion in the ALS-discordant twin pair 421 and 422 (see Supplementary Material), which have been previously reported [55]. However, we have not been able to follow-up these and other twins to determine whether there may be delayed onset in the "unaffected" co-twin. In light of this, it would be worthwhile to re-evaluate earlier twin studies. In addition, functional validation of the variants reported herein is warranted, a lack of which is acknowledged as a limitation.

Acknowledgements We are grateful to the study participants and The Leverhulme Trade Charities Trust for a bursary to NV and The Dowager Countess Eleanor Peel Trust for generously supporting the work (JN).

\section{Compliance with ethical standards}

Conflict of interest The authors declare that they have no conflict of interest.

Publisher's note: Springer Nature remains neutral with regard to jurisdictional claims in published maps and institutional affiliations.

\section{References}

1. van Dongen J, Slagboom PE, Draisma HHM, Martin NG, Boomsma DI. The continuing value of twin studies in the omics era. Nat Rev Genet. 2012;13:640-53.

2. Hilker R, Helenius D, Fagerlund B, Skytthe A, Christensen K, Werge TM, et al. Heritability of schizophrenia and schizophrenia spectrum based on the Nationwide Danish Twin Register. Biol Psychiatry. 2018;83:492-8.

3. Al-Chalabi A, Fang F, Hanby MF, Leigh PN, Shaw CE, Ye W, et al. An estimate of amyotrophic lateral sclerosis heritability using twin data. J Neurol Neurosurg Psychiatry. 2010;81: 1324-6.

4. Colvert E, Tick B, McEwen F, Stewart C, Curran SR, Woodhouse E, et al. Heritability of autism spectrum disorder in a UK population-based twin sample. JAMA Psychiatry. 2015;72: 415-23.

5. Mataix-Cols D, Isomura K, Pérez-Vigil A, Chang Z, Rück C, Larsson KJ, et al. Familial risks of Tourette syndrome and chronic Ticdisorders. JAMA Psychiatry. 2015;72:787. 
6. Ketelaar ME, Hofstra EMW, Hayden MR. What monozygotic twins discordant for phenotype illustrate about mechanisms influencing genetic forms of neurodegeneration. Clin Genet. 2012;81:325-33.

7. Dal GM, Ergüner B, Sağıroğlu MS, Yüksel B, Onat OE, Alkan C, et al. Early postzygotic mutations contribute to de novo variation in a healthy monozygotic twin pair. J Med Genet. 2014;51:455-9.

8. Kong A, Frigge ML, Masson G, Besenbacher S, Sulem P, Magnusson $\mathrm{G}$, et al. Rate of de novo mutations and the importance of father's age to disease risk. Nature. 2012;488:471-5.

9. Campbell CD, Chong JX, Malig M, Ko A, Dumont BL, Han L, et al. Estimating the human mutation rate using autozygosity in a founder population. Nat Genet. 2012;44:1277-81.

10. Freed D, Stevens EL, Pevsner J. Somatic mosaicism in the human genome. Genes (Basel). 2014;5:1064-94.

11. Francioli LC, Polak PP, Koren A, Menelaou A, Chun S, Renkens I, et al. Genome-wide patterns and properties of de novo mutations in humans. Nat Genet. 2015;47:822-6.

12. Acuna-Hidalgo R, Bo T, Kwint MP, van de Vorst M, Pinelli M, Veltman JA, et al. Post-zygotic point mutations are an underrecognized source of de novo genomic variation. Am J Hum Genet. 2015;97:67-74.

13. Robertson SP, Thompson S, Morgan T, Holder-Espinasse M, Martinot-Duquenoy V, Wilkie AOM, et al. Postzygotic mutation and germline mosaicism in the otopalatodigital syndrome spectrum disorders. Eur J Hum Genet. 2006;14:549-54.

14. Vadlamudi L, Dibbens LM, Lawrence KM, Iona X, McMahon $\mathrm{JM}$, Murrell W, et al. Timing of de novo mutagenesis - a twin study of sodium-channel mutations. N Engl J Med. 2010;363: 1335-40.

15. Lindhurst MJ, Sapp JC, Teer JK, Johnston JJ, Finn EM, Peters K, et al. A mosaic activating mutation in AKT1 associated with the proteus syndrome. N Engl J Med. 2011;365:611-9.

16. Ehli EA, Abdellaoui A, Hu Y, Hottenga JJ, Kattenberg M, van Beijsterveldt $\mathrm{T}$, et al. De novo and inherited CNVs in MZ twin pairs selected for discordance and concordance on attention problems. Eur J Hum Genet. 2012;20:1037-43.

17. Tang J, Fan Y, Li H, Xiang Q, Zhang D-F, Li Z, et al. Wholegenome sequencing of monozygotic twins discordant for schizophrenia indicates multiple genetic risk factors for schizophrenia. J Genet Genom. 2017;44:295-306.

18. Kuhlenbäumer G, Hullmann J, Appenzeller S. Novel genomic techniques open new avenues in the analysis of monogenic disorders. Hum Mutat. 2011;32:144-51.

19. McKenna A, Hanna M, Banks E, Sivachenko A, Cibulskis K, Kernytsky A, et al. The Genome Analysis Toolkit: a MapReduce framework for analyzing next-generation DNA sequencing data. Genome Res. 2010;20:1297-303.

20. Wang K, Li M, Hakonarson H. ANNOVAR: functional annotation of genetic variants from high-throughput sequencing data. Nucleic Acids Res. 2010;38:e164-e164.

21. Kumar P, Henikoff S, Ng PC. Predicting the effects of coding non-synonymous variants on protein function using the SIFT algorithm. Nat Protoc. 2009;4:1073-81.

22. Adzhubei IA, Schmidt S, Peshkin L, Ramensky VE, Gerasimova A, Bork P, et al. A method and server for predicting damaging missense mutations. Nat Methods. 2010;7:248-9.

23. Schwarz JM, Rödelsperger C, Schuelke M, Seelow D. MutationTaster evaluates disease-causing potential of sequence alterations. Nat Methods. 2010;7:575-6.

24. Davydov EV, Goode DL, Sirota M, Cooper GM, Sidow A, Batzoglou S. Identifying a high fraction of the human genome to be under selective constraint using GERP ++ . PLoS Comput Biol. 2010;6:e1001025.

25. Wang K, Li M, Hadley D, Liu R, Glessner J, Grant SFA, et al. PennCNV: an integrated hidden Markov model designed for high-resolution copy number variation detection in whole-genome SNP genotyping data. Genome Res. 2007;17:1665-74.

26. Labrie V, Buske OJ, Oh E, Jeremian R, Ptak C, Gasiūnas G, et al. Lactase nonpersistence is directed by DNA-variation-dependent epigenetic aging. Nat Struct Mol Biol. 2016;23:566-73.

27. Beicht S, Strobl-Wildemann G, Rath S, Wachter O, Alberer M, Kaminsky E, et al. Next generation sequencing as a useful tool in the diagnostics of mosaicism in Alport syndrome. Gene. 2013;526:474-7.

28. Petrovski S, Wang Q, Heinzen EL, Allen AS, Goldstein DB. Genic intolerance to functional variation and the interpretation of personal genomes. PLoS Genet. 2013;9:e1003709.

29. Levinson DF, Duan J, Oh S, Wang K, Sanders AR, Shi J, et al. Copy number variants in schizophrenia: confirmation of five previous findings and new evidence for $3 q 29$ microdeletions and VIPR2 duplications. Am J Psychiatry. 2011;168:302-16.

30. Baranzini SE, Mudge J, van Velkinburgh JC, Khankhanian P, Khrebtukova I, Miller NA, et al. Genome, epigenome and RNA sequences of monozygotic twins discordant for multiple sclerosis. Nature. 2010;464:1351-6.

31. Petersen B-S, Spehlmann ME, Raedler A, Stade B, Thomsen I, Rabionet $\mathrm{R}$, et al. Whole genome and exome sequencing of monozygotic twins discordant for Crohn's disease. BMC Genomics. 2014;15:564.

32. Magne F, Serpa R, Van Vliet G, Samuels ME, Deladoëy J. Somatic mutations are not observed by exome sequencing of lymphocyte DNA from monozygotic twins discordant for congenital hypothyroidism due to thyroid dysgenesis. Horm Res Paediatr. 2015;83:79-85.

33. Meltz Steinberg K, Nicholas TJ, Koboldt DC, Yu B, Mardis E, Pamphlett R. Whole genome analyses reveal no pathogenetic single nucleotide or structural differences between monozygotic twins discordant for amyotrophic lateral sclerosis. Amyotroph Lateral Scler Front Degener. 2015;16:385-92.

34. Cai L, Yuan W, Zhang Z, He L, Chou K-C. In-depth comparison of somatic point mutation callers based on different tumor nextgeneration sequencing depth data. Sci Rep. 2016;6:36540.

35. Cecchinato A, Chessa S, Ribeca C, Cipolat-Gotet C, Bobbo T, Casellas $\mathrm{J}$, et al. Genetic variation and effects of candidate-gene polymorphisms on coagulation properties, curd firmness modeling and acidity in milk from Brown Swiss cows. Animal. 2015; 9:1104-12.

36. Nickles D, Madireddy L, Yang S, Khankhanian P, Lincoln S, Hauser SL, et al. In depth comparison of an individual's DNA and its lymphoblastoid cell line using whole genome sequencing. BMC Genomics. 2012;13:477.

37. Vitucci D, Di Giorgio A, Napolitano F, Pelosi B, Blasi G, Errico $\mathrm{F}$, et al. Rasd2 modulates prefronto-striatal phenotypes in humans and 'schizophrenia-like behaviors' in mice. Neuropsychopharmacology. 2016;41:916-27.

38. Potash JB, Zandi PP, Willour VL, Lan T-H, Huo Y, Avramopoulos $\mathrm{D}$, et al. Suggestive linkage to chromosomal regions $13 \mathrm{q} 31$ and $22 \mathrm{q} 12$ in families with psychotic bipolar disorder. Am J Psychiatry. 2003;160:680-6.

39. Liu Y-L, Fann CS-J, Liu C-M, Chen WJ, Wu J-Y, Hung S-I, et al. RASD2, MYH9, and CACNG2 genes at chromosome 22q12 associated with the subgroup of schizophrenia with non-deficit in sustained attention and executive function. Biol Psychiatry. 2008;64:789-96.

40. Bertelsen B, Stefánsson H, Riff Jensen L, Melchior L, Mol Debes $\mathrm{N}$, Groth C, et al. Association of AADAC deletion and Gilles de la Tourette syndrome in a large European Cohort. Biol Psychiatry. 2016;79:383-91.

41. Yuan L, Zheng W, Yang Z, Deng X, Song Z, Deng H. Association of the AADAC gene and Tourette syndrome in a Han Chinese cohort. Neurosci Lett. 2018;666:24-7. 
42. Ebstein RP, Monakhov M, Lai PS, Chew SH. CD38 gene expression and human personality traits: inverse association with novelty seeking. Messenger. 2014;3:72-7.

43. Higashida H, Yokoyama S, Huang J-J, Liu L, Ma W-J, Akther S, et al. Social memory, amnesia, and autism: brain oxytocin secretion is regulated by NAD + metabolites and single nucleotide polymorphisms of CD38. Neurochem Int. 2012;61:828-38.

44. Fallon J, Reid S, Kinyamu R, Opole I, Opole R, Baratta J, et al. In vivo induction of massive proliferation, directed migration, and differentiation of neural cells in the adult mammalian brain. Proc Natl Acad Sci. 2000;97:14686-91.

45. Akbarian S, Bunney WE, Potkin SG, Wigal SB, Hagman JO, Sandman CA, et al. Altered distribution of nicotinamide-adenine dinucleotide phosphate-diaphorase cells in frontal lobe of schizophrenics implies disturbances of cortical development. Arch Gen Psychiatry. 1993;50:169-77.

46. Lavedan C, Licamele L, Volpi S, Hamilton J, Heaton C, Mack K, et al. Association of the NPAS3 gene and five other loci with response to the antipsychotic iloperidone identified in a whole genome association study. Mol Psychiatry. 2009;14:804-19.

47. Chen J, Calhoun VD, Perrone-Bizzozero NI, Pearlson GD, Sui J, $\mathrm{Du} \mathrm{Y}$, et al. A pilot study on commonality and specificity of copy number variants in schizophrenia and bipolar disorder. Transl Psychiatry. 2016;6:e824-e824.

48. Antonacci F, Dennis MY, Huddleston J, Sudmant PH, Steinberg KM, Rosenfeld JA, et al. Palindromic GOLGA8 core duplicons promote chromosome $15 \mathrm{q} 13.3$ microdeletion and evolutionary instability. Nat Genet. 2014;46:1293-302.

49. Florio M, Albert M, Taverna E, Namba T, Brandl H, Lewitus E, et al. Human-specific gene ARHGAP11B promotes basal progenitor amplification and neocortex expansion. Science. 2015; 347:1465-70.

50. Dennis MY, Eichler EE. Human adaptation and evolution by segmental duplication. Curr Opin Genet Dev. 2016;41:44-52.

51. Ohi K, Hashimoto R, Nakazawa T, Okada T, Yasuda Y, Yamamori $\mathrm{H}$, et al. The p250GAP gene is associated with risk for schizophrenia and schizotypal personality traits. PLoS ONE. 2012;7:e35696.

52. Schuster S, Rivalan M, Strauss U, Stoenica L, Trimbuch T, Rademacher $\mathrm{N}$, et al. NOMA-GAP/ARHGAP33 regulates synapse development and autistic-like behavior in the mouse. Mol Psychiatry. 2015;20:1120-31.

53. Hashimoto R, Yoshida M, Ozaki N, Yamanouchi Y, Iwata N, Suzuki T, et al. A missense polymorphism (H204R) of a Rho GTPase-activating protein, the chimerin 2 gene, is associated with schizophrenia in men. Schizophr Res. 2005;73:383-5.

54. Wong EHM, So H-C, Li M, Wang Q, Butler AW, Paul B, et al. Common variants on $\mathrm{Xq} 28$ conferring risk of schizophrenia in Han chinese. Schizophr Bull. 2014;40:777-86.

55. Pamphlett R, Cheong PL, Trent RJ, Yu B. Can ALS-associated C9orf72 repeat expansions be diagnosed on a blood DNA test Alone? PLoS ONE. 2013;8:e70007. 